\title{
Arbuscular mycorrhizal inoculation and super phosphate application influence plant growth and yield of Capsicum annuum
}

\author{
A. $\operatorname{Tanwar}^{1 *}$, A. Aggarwal, ${ }^{1}$ N. $\operatorname{Kadian}^{1}$ and A. Gupta ${ }^{2}$ \\ ${ }^{1}$ Mycology and Plant Pathology Laboratory, Department of Botany, Kurukshetra University, Kurukshetra-136119, Haryana, \\ India *e-mail: anjutanwarbotany@gmail.com, Mob.: *+91-9729469510. ²Department of Botany, University College, \\ Kurukshetra University, Kurukshetra-136119, Haryana, India
}

\begin{abstract}
The effect of two arbuscular mycorrhizal fungi $[G$. mosseae $(\mathrm{G})$ and A. laevis (A)] with P. fluorescence (Pf) in the presence of super phosphate $(\mathrm{P})$ fertilization on growth and yield of bell pepper (Capsicum annuum var. California Wonder) was evaluated in pots under greenhouse conditions, in a completely randomized design with four levels of phosphorus fertilizer [F0-without P, F1-0.200 pot $^{-1}$ (half of the recommended dose), F3-0.400 g pot $^{-1}$ (recommended dose) and F4-0.800 g pot $^{-1}$ (double the recommended dose)] having six different combinations of bioinoculants. Inoculation of bioinoclulants with F1 increased plant growth and nutrition to an acceptable level with AM fungi in combination with P. fluorescens. Application of higher dose of $\mathrm{P}$ fertilizer markedly decreased all the growth parameters. The prevalence of AM colonization was highest in $\mathrm{G}+\mathrm{A}+\mathrm{Pf}$ with $\mathrm{F}$. Similarly highest yield was recorded for the treatment involving multi inoculation of $\mathrm{G}+\mathrm{A}+\mathrm{Pf}$ in the treatment of $\mathrm{F} 1$ followed by dual inoculation of $\mathrm{G}+\mathrm{Pf}$ in $\mathrm{F} 0$ plants. Thus this finding suggests the application of efficient bioinoculants $(\mathrm{G}+\mathrm{A}+\mathrm{Pf})$ along with right dose of $\mathrm{P}$ fertilizer (half of the recommended $\mathrm{P}$ ) during seedling transplantation to increase overall growth and yield performance of bell pepper and could be considered as a sustainable substitute to higher phosphorus fertilizer for bell pepper cultivation.
\end{abstract}

Keywords: phosphorus fertilizer, fruit yield, Pseudomonas fluorescens, bell pepper, endomycorrhizal fungi 


\section{Introduction}

Horticultural crop species such as bell pepper (Capsicum annuum L.) is one of the most valuable crops of India. It has nutritive and medicinal value as is a good source of vitamin A, C, E and also has antioxidants property. Phosphorus is known to be one of the most essential elements for plant growth and development after nitrogen. The phosphorus status of Kurukshetra district, Haryana where the experiment was performed is low i.e., $0-24.7 \mathrm{~kg} \mathrm{ha}^{-1}$ which is not suitable for bell pepper cultivation and needs an external source of phosphorus fertilizer (Tanwar et al., 2012).

Due to the low cost of the phosphate fertilizers and their easy availablility, farmers usually over-fertilize their field, rather than risk under-fertilizing and suffering yield lost. The available phosphorus in the soil is present in very low concentration as large amount is bound in its inorganic form. On the other hand the availability of organic forms e.g., Phytate is actually controlled by its mineralization rate in the soil. The mobility of this element is very slow in the soil and can not be replenished due to rapid uptake by plants roots. That results in the formation of phosphorus depleted zones near the contact area of roots and soil with in rhizosphere. Soluble phosphorus can be released from insoluble phosphates by solublization reaction involving rhizospheric microorganisms (Kapoor et al., 1989).

Current strategies adopted for fruit and vegetable production use fertilizer application regimes that are aimed to reach maximum volume or weight of yield, sometimes neglecting the aspects of quality (Oke et al., 2005). Therefore, the plants need an assisting system which could extend beyond the depletion zones and help to absorb the phosphorus from a wider area by developing an extended network around root system. Recently the employment of beneficial microorganisms has gained popularity (Parkash et al., 2011). The use of organic fertilizer and microbial inoculants or biofertilizers represents a sustainable alternative to high input of chemical fertilizers used in the conventional production systems (Kennedy et al., 2004).

The importance of Arbuscular mycorrhizal (AM) fungi in natural and semi natural ecosystems is commonly accepted by improved plant productivity and diversity as well as increased plant resistance against biotic and abiotic stresses (Smith and Read, 2008). The fungus contributes greatly to phosphorus uptake (Schnepf et al., 2009), besides the uptake of other ions. The primary advantage of mycorrhizal hyphae in P uptake is the ability of hyphae to extend deeper in the soil beyond $\mathrm{P}$ depletion zone of the roots (Jacobsen, 1995). It has been reported that plant dependency to mycorrhizal fungi depends on the level of soil fertility and receptivity of soil to inoculants (Covacevich and Echeverria, 2008).

Consortium use of AM fungi along with some rhizobacteria to enhance quantity and quality of plant production in agriculture is relatively recent technology. Among the several free-living microorganisms, plant growth-promoting rhizobacteria (PGPR) have received special attention, due to their beneficial effect on plant growth promotion by enhancing mineral nutrition (Gamalero et al., 2004) and synthesis of phytohormones (Gamalero et al., 2008). In particular, Pseudomonas flourscens is considered as an important member of rhizosphere organism community. The positive effect of Pseudomonas inoculation on plant growth has been reported in many research trials (Constantino et al., 2008, Tanwar et al., 2011; Prasad et al., 2012; Tanwar et al., 2012; Tanwar and Aggarwal, 2013; Eslamyan et al., 2013).

Hence, this experiment was conducted in a greenhouse condition to compare the efficiency of different concentrations of super phosphate fertilizer in association with AM fungi and Pseudomonas fluorescens on growth, nutrition and yield of Capsicum annuum with an aim to reduce the application of chemical fertilizer for sustainable system. 


\section{Materials and Methods}

\subsection{Isolation and identification of AM fungi}

Dominant AM fungi were isolated from the rhizosphere soil of field grown bell pepper plants by 'wet sieving and decanting technique' of Gerdemann and Nicolson (1963). The isolated spores were identified using keys of Schenck and Perez (1990) and Glomus mosseae Nicol. and Gerd. and Acaulospora laevis Gerd. and Trappe were found to be the most dominant AM fungal strains.

\subsection{Experimental design}

The experiment was conducted in a $4 \times 6$ factorial in a completely randomized design employing four levels of super phosphate fertilizer (F0-without P, F1-0.200 $\mathrm{g} \mathrm{pot}^{-1}, \mathrm{~F} 2-0.500 \mathrm{~g} \mathrm{pot}^{-1}$ and F3-0.800 $\mathrm{g} \mathrm{pot}^{-1}$ ) and six levels of different bioinoculant combinations i.e. control (C), G. mosseae (G), A. laevis (A), G. mosseae + P. fluorescens $(\mathrm{G}+\mathrm{Pf})$, A. laevis + P. fluorescens $(\mathrm{A}+\mathrm{Pf})$, G. mosseae + A. laevis + P. fluorescens $(\mathrm{G}+\mathrm{A}+\mathrm{Pf})$ with five replicates of each.

\subsection{Bioinoculant preparation}

The starter inoculum of each selected dominant AM fungus was raised by funnel technique of Menge and Timmer (1982) using Maize as host for three months. The inoculum of $P$. fluorescens (MTCC No. 103) was obtained from Institute of Microbial Technology, Chandigarh, India and cultured in a nutrient broth medium incubated at $32^{\circ} \mathrm{C}$ for 48 hours to obtain a concentration of $1 \times 10^{9}$ colony forming units (cfu) $\mathrm{mL}^{-1}$.

\subsection{Experimental setup}

The soil used in the study has sand- $64.2 \%$, silt$21.81 \%$, clay-3.90\%, pH-6.8 $\pm 0, \mathrm{EC}-0.25 \mathrm{dS}^{-1} / \mathrm{m}$, organic carbon $-0.40 \%$, total $\mathrm{N}-0.042 \%, \mathrm{P}-7.30 \mathrm{~kg}$ 'acre, $\mathrm{K}-88 \mathrm{~kg}^{-1}$ acre and $\mathrm{S}-14.80 \mathrm{ppm}$. The seeds of bell pepper (Capsicum annuит var. California Wonder) were germinated using a shallow tray containing soil: sand (3:1) which was autoclaved at $120^{\circ} \mathrm{C}$ for two consecutive days before it is used for germination. Experimental pots were filled with $2 \mathrm{~kg}$ sterilized soil followed by AM inoculation. For single inoculation $10 \%$, i.e., $200 \mathrm{~g}$ of air dried AM inoculum (G. mosseae and $A$. laevis) containing around 865 spores and $80 \%$ colonized root segments of trap host Maize were used. For double inoculation, $100 \mathrm{~g}$ inoculum and for triple $65 \mathrm{~g}$ of AM fungi inoculum was used. Fifteen days after germination, single seedling was transplanted to each pot. Similarly, in treatment with $P$. fluorescens, bell pepper roots were first dipped in conidial suspension of $P$. fluorescens for five minutes and planted in each pot. Control pots without microbial inoculation were also maintained.

Four levels of solid super phosphate fertilizers $(0 \mathrm{~g}, 0.200 \mathrm{~g}, 0.400 \mathrm{~g}$ and $0.800 \mathrm{~g})$ were added as a basal dressing in pots one week before microbial inoculation. Plants were maintained in greenhouse, watered daily to maintain the moisture approximately at $60 \%$ water holding capacity of the soil and also fertilized with Hoagland nutrient solution except for phosphorus source, after every 15 days.

\subsection{Harvest and analysis}

Plant development was assessed by measuring plant height $(\mathrm{cm})$, shoot fresh and dry weight $(\mathrm{g})$ and root fresh and dry weight $(\mathrm{g})$. Plant height was measured from soil surface to the growing tip of the plant, after that, plants were harvested at the fruiting stage (120 days) by removing the whole plant completely from the pot. Number of days for flowering and fruit setting was also recorded upto 120 days. Subsequently plants were divided into shoots and roots and further into fruits. Yield of plant was assessed by counting number of fruits per plant and by evaluating the fresh weight of fruits. The plant shoot was cut into pieces of approximately $10 \mathrm{~cm}$ size and fresh weight was taken. Similarly fresh weight of roots was taken for each treatment. Dry matter was estimated by keeping the plant in oven at $60{ }^{\circ} \mathrm{C}$ then weighed to get the dry matter for shoot as well as root. Stomatal conductance 
by using porometer (AP4-Delta T devices, cambridge, UK) and chlorophyll content by using Arnon's method (1949). Photosynthesis was measured in $\mathrm{mg} \mathrm{CO}_{2} \mathrm{~m}^{-2} \mathrm{~s}^{-1}$ using portable Infra Red Gas Analyzer, CIRCAS-I, PP Systems, UK.

Percent root colonization was assessed by cleaning the roots with $10 \% \mathrm{KOH}$ followed by staining with $0.01 \%$ trypan blue (Phillips and Hayman, 1970). The AM spore extraction was done using the procedure of Gerdemann and Nicolson (1963). The phosphorus content in shoot and root was determined by Vanadomolybdo-phosphoric acid yellow colour method, in nitric acid system outlined by Jackson (1973), which is actually based on the yellow colour of the unreduced Vanado-molybdo-phosphoric heteropoly complex. Total nitrogen was calculated by Kjeldahl method (Kelplus nitrogen estimation system, supra-LX, Pelican Equipments, Chennai, India).

\subsection{Statistical analysis}

The experimental data was analyzed using Analysis of Variance (ANOVA), followed by post hoc test using the Statistical Package for Social Sciences (ver. 11.5, Chicago, Ill.). Means were then ranked at $p \leq 0.05$ level of significance using Duncan's Multiple Range Test for comparison.

\section{Results and Discussion}

All different bioinoculats and/or phosphorus fertilization has significant effect on all measured plant growth parameters. However, the level to which growth is enhanced varied between the bio inoculants and $\mathrm{P}$ fertilizer doses.

\subsection{Effect on mycorrhization}

All the plants inoculated with AM fungi showed mycorrhizal colonization that was characterized by the presence of extramatrical hyphae, intraradical hyphae, arbuscules and vesicles. However, mycorrhizal colonization, arbuscule and vesicle formation significantly decreased with the increase in fertilizer application rate. Prominent vesicles of G. mosseae and A. laevis had formed within the root cell, which shows the affinity of the fungus with Capsicum annuum. Similar trend was observed with mycorrhizal spore number also, but there was no positive correlation between mycorrhizal spore number and colonization rate.

After 120 days of inoculation sole application of $G$. mosseae produced maximum spore number $(93.8 \pm 6)$, while the application of the same AM fungi along with $P$. fluorescens achieved maximum root colonization $(97.62 \pm 3.3)$ at F1 (Table-1). These results are in close conformity with those of other that the co-inoculation of AM fungi with P. fluorescens, or other rhizobacteria increased the capacity of AM fungi to colonize the plant roots (Boer et al., 2005; Tanwar et al., 2012). The high rate of $\mathrm{P}$ fertilizer application i.e., $\mathrm{F} 2$ and F3, lead to antagonistic inhibition of mycorrhizal colonization whereas in lower dose, mycorrhiza was able to increase the root colonization significantly. Similar finding were reported earlier also that the colonization potential of AM fungi decreases with increasing $P$ concentration in the soil (Prasad et al., 2012).

\subsection{Effect on plant growth performance}

The effect of $\mathrm{P}$ on plant growth performance was contrasting. Some parameters are enhanced with increasing concentration whereas other parameters did not show any stimulatory effect. There were significant differences in plant height, shoot and root fresh and dry weight and root length between inoculated and control plants. As shown in Table 2, G. mosseae colonized plants performed consistently better than A. laevis alone and also in combination with $P$. fluorescens. In our earlier study also we found $G$. mosseae as much compatible strain than the other AM strain used for red bell pepper production (F1 hybrid, Indam Mamatha) (Tanwar and Aggarwal, 2013). 
Table 1. Effect of soil inoculation with AM fungi, P. fluorescens and super phosphate on mycorrhization of bell pepper plants

\begin{tabular}{|c|c|c|c|}
\hline $\begin{array}{l}\text { Super phosphate } \\
\text { concentration (g/pot) }\end{array}$ & $\begin{array}{l}\text { Parameters } \rightarrow \\
\text { Treatments } \downarrow\end{array}$ & $\begin{array}{l}\text { AM spore number } \\
/ 10 \text { g soil }\end{array}$ & $\begin{array}{l}\text { AM root } \\
\text { colonization (\%) }\end{array}$ \\
\hline $\begin{array}{l}\text { F0 } \\
\text { Without fertilizer }\end{array}$ & $\begin{array}{l}\text { Control } \\
G \\
A \\
G+P f \\
A+P f \\
G+A+P f\end{array}$ & $\begin{array}{l}11.20 \pm 2.33 \mathrm{~g} \\
70.8 \pm 6.1 \mathrm{bc} \\
65.4 \pm 5.64 \mathrm{bc} \\
52.8 \pm 4.6 \mathrm{~d} \\
67.6 \pm 3.64 \mathrm{bc} \\
69.7 \pm 4.77 \mathrm{bc}\end{array}$ & $\begin{array}{l}15.77 \pm 3.38 \mathrm{~g} \\
86.71 \pm 4.61 \mathrm{ab} \\
88.22 \pm 7.01 \mathrm{ab} \\
74.91 \pm 7.91 \mathrm{~b} \\
74.98 \pm 4.97 \mathrm{~b} \\
74.27 \pm 3 \mathrm{~b}\end{array}$ \\
\hline $\begin{array}{l}\text { F1 } \\
\text { Half recommended } \\
(0.200 \mathrm{~g} / \text { pot })\end{array}$ & $\begin{array}{l}\text { Control } \\
G \\
A \\
G+P f \\
A+P f \\
G+A+P f\end{array}$ & $\begin{array}{l}24.0 \pm 1.58 \mathrm{f} \\
93.8 \pm 6 \mathrm{a} \\
76.8 \pm 3.4 \mathrm{~b} \\
62.6 \pm 4.5 \mathrm{c} \\
51.4 \pm 4.3 \mathrm{~d} \\
80.2 \pm 3.5 \mathrm{ab}\end{array}$ & $\begin{array}{l}11.62 \pm 2.42 \mathrm{~g} \\
93.98 \pm 6.13 \mathrm{a} \\
94.29 \pm 6.82 \mathrm{a} \\
85.68 \pm 7.2 \mathrm{ab} \\
86.44 \pm 2.5 \mathrm{ab} \\
97.62 \pm 3.3 \mathrm{a}\end{array}$ \\
\hline $\begin{array}{l}\text { F2 } \\
\text { Recommended } \\
(0.400 \mathrm{~g} / \text { pot }\end{array}$ & $\begin{array}{l}\text { Control } \\
G \\
A \\
G+P f \\
A+P f \\
G+A+P f\end{array}$ & $\begin{array}{l}20.0 \pm 4 \mathrm{f} \\
76.2 \pm 3.7 \mathrm{~b} \\
62.4 \pm 3.2 \mathrm{c} \\
44.0 \pm 2.9 \mathrm{de} \\
33.0 \pm 3.67 \mathrm{e} \\
56.4 \pm 2.7 \mathrm{~d} \\
\end{array}$ & $\begin{array}{l}22.14 \pm 2.2 \mathrm{f} \\
89.28 \pm 2.7 \mathrm{ab} \\
77.30 \pm 3.1 \mathrm{~b} \\
73.52 \pm 4.7 \mathrm{~b} \\
69.32 \pm 6.9 \mathrm{c} \\
79.10 \pm 4.4 \mathrm{~b}\end{array}$ \\
\hline $\begin{array}{l}\text { F3 } \\
\text { Double } \\
\text { recommended } \\
(0.800 \mathrm{~g} / \text { pot })\end{array}$ & $\begin{array}{l}\text { Control } \\
G \\
A \\
G+P f \\
A+P f \\
G+A+P f\end{array}$ & $\begin{array}{l}19.3 \pm 2.4 \mathrm{f} \\
19.0 \pm 1.87 \mathrm{f} \\
21.8 \pm 1.9 \mathrm{f} \\
17.6 \pm 2.1 \mathrm{fg} \\
16.8 \pm 2.6 \mathrm{fg} \\
23.4 \pm 4.87 \mathrm{f} \\
\end{array}$ & $\begin{array}{l}14.45 \pm 4.3 \mathrm{~g} \\
30.79 \pm 9.2 \mathrm{e} \\
27.00 \pm 4.41 \mathrm{e} \\
45.02 \pm 6.34 \mathrm{~d} \\
30.81 \pm 4.6 \mathrm{e} \\
26.66 \pm 3.3 \mathrm{e} \\
\end{array}$ \\
\hline $\begin{array}{r}\text { ANOVA }\left(\mathrm{F}_{5}, 96\right) \\
\left(\mathrm{F}_{3}, 96\right) \\
\left(\mathrm{F}_{15}, 96\right)\end{array}$ & $\begin{array}{l}\text { Treatments }(\mathrm{t}) \\
\text { Fertilizer }(\mathrm{F}) \\
\mathrm{t} \times \mathrm{F}\end{array}$ & $\begin{array}{l}401.75 \\
543.65 \\
61.39 \\
\end{array}$ & $\begin{array}{l}470.57 \\
374.56 \\
30.31 \\
\end{array}$ \\
\hline
\end{tabular}

Each value is a mean of five replicates, \pm : standard deviation, G: Glomus mosseae, A: Acaulospora laevis, Pf: Pseudomonas fluorescens, F: Super phosphate fertilization. Means followed by same letter within a column are not significantly different over one another (Duncan's Multiple Range Test, $p \leq 0.05$ ).

The dual inoculation of Pf with either of the mycorrhizal fungi showed stimulatory effects by improving plant growth in comparison to uninoculated control. This increase can be due to absorbance of more P from the soil and its accumulation towards shoots, resulting in increased plant height $(67.24 \pm 0.53)$ in $\mathrm{G}+\mathrm{Pf}$ and maximum shoot fresh $(28.16 \pm 1.12)$ and dry weight $(6.08 \pm 2.14)$ in $\mathrm{G}+\mathrm{A}+\mathrm{Pf}$ at $\mathrm{F} 1$. However, $\mathrm{G}+\mathrm{A}+\mathrm{Pf}$ at F2 showed highest root length $(39.62 \pm 0.86)$, root fresh $(9.51 \pm 1.02)$ and dry weight $(2.73 \pm 0.38)$. Gamalero et al. (2004) also documented that the presence of PGRP (e.g. Attinomyces, Pseudomonas spp., Bacillus spp.) in mycorrhizal inoculum can synergistically improve tomato growth. Our finding is also in agreement with that of Anguilera-Gomez et al. (1999) that AM fungi increased leaf number,leaf 
area shoot, root and fruit mass at lower concentrations of $\mathrm{P}$ fertilizer as compared to the heavy P fertilization doses that increased the dry matter of the plant but at the same time decreases mycorrhizal colonization of the host roots.

\subsection{Effect on photosynthetic rate}

It seems that higher dose of phosphorus does not play an important role in enhancement of leaf chlorophyll, photosynthesis and stomatal conductance (Table 3). Mycorrhizal inoculation of plants does increase the chlorophyll content, phosyntesis and stomatal conductance as compared to the uninoculated control. Increased chlorophyll content on inoculation with AM fungi is also reported by Qiao et al. (2011). The highest increase in photosynthetic rate, that was measured as chlorophyll a (123.06 \pm 2.4$)$, chlorophyll b (448.5 \pm 4.75$)$, photosynthesis $(51.06 \pm 2.96)$ and stomatal conductance both on the upper $(0.272 \pm 0.0084)$ and lower $(0.834 \pm 0.009)$ side was markedly enhanced in $\mathrm{G}+\mathrm{A}+\mathrm{Pf}$ of $\mathrm{F} 1$.

Table 2. Effect of soil inoculation with AM fungi, P. fluorescens and super phosphate on plant growth response of bell pepper

\begin{tabular}{|c|c|c|c|c|c|c|c|}
\hline \multirow{2}{*}{$\begin{array}{l}\text { Super } \\
\text { phosphate } \\
\text { concentration } \\
\text { (g/pot) }\end{array}$} & \multirow{2}{*}{$\begin{array}{l}\text { Parameters } \\
\rightarrow \\
\text { Treatments } \\
\downarrow\end{array}$} & \multirow{2}{*}{$\begin{array}{l}\text { Increase in } \\
\text { plant height } \\
\text { (cm) }\end{array}$} & \multicolumn{2}{|c|}{ Above ground weight (g) } & \multirow{2}{*}{$\begin{array}{l}\text { Root length } \\
(\mathrm{cm})\end{array}$} & \multicolumn{2}{|c|}{ Root weight (g) } \\
\hline & & & Fresh & Dry & & Fresh & Dry \\
\hline F0 & Control & $21.28 \pm 0.94 \mathrm{f}$ & $3.65 \pm 0.17 \mathrm{f}$ & $1.66 \pm 0.08 \mathrm{f}$ & $9.260 \pm 0.33 \mathrm{e}$ & $0.72 \pm 0.05 f$ & $0.27 \pm 0.02 \mathrm{f}$ \\
\hline Without & G & $54.90 \pm 2.1 \mathrm{~b}$ & $13.7 \pm 0.58 \mathrm{bc}$ & $2.82 \pm$ & $0.26 \mathrm{c}$ & $35 \mathrm{~cd}$ & $24 \mathrm{de}$ \\
\hline \multirow[t]{4}{*}{ fertilizer } & A & $51.24 \pm 0.78 \mathrm{~b}$ & $10.3 \pm 0.3 \mathrm{c}$ & $2.73 \pm 0.08 \mathrm{~d}$ & $15.88 \pm 0.41 \mathrm{c}$ & $4.30 \pm 0.36 \mathrm{de}$ & $0.58 \pm 0.01 \mathrm{e}$ \\
\hline & $\mathrm{G}+\mathrm{Pf}$ & $55.86 \pm 2.26 \mathrm{~b}$ & $7.36 \pm 0.21 \mathrm{~d}$ & $2.60 \pm 0.05 \mathrm{~d}$ & $17.12 \pm 0.28 \mathrm{c}$ & $3.20 \pm 0.33 \mathrm{e}$ & $0.32 \pm 0.02 \mathrm{e}$ \\
\hline & $\mathrm{A}+\mathrm{Pf}$ & $34.12 \pm 0.71 d$ & $8.30 \pm 0.25 \mathrm{de}$ & $2.70 \pm 0.05 d$ & $20.00 \pm 0.46 \mathrm{bc}$ & $3.60 \pm 0.25 \mathrm{de}$ & $0.74 \pm 0.02 \mathrm{de}$ \\
\hline & $\mathrm{G}+\mathrm{A}+\mathrm{Pf}$ & $45.90 \pm 0.86 c$ & $13.7 \pm 0.33 \mathrm{bc}$ & $2.80 \pm 0.13 \mathrm{~d}$ & $12.28 \pm 0.59 \mathrm{~d}$ & $1.87 \pm 0.09 \mathrm{ef}$ & $0.45 \pm 0.03 \mathrm{~d}$ \\
\hline F1 & Control & $20.48 \pm 2.33 \mathrm{f}$ & $5.86 \pm 0.24 \mathrm{e}$ & $1.86 \pm 0.34 \mathrm{e}$ & $10.40 \pm 1.17 \mathrm{~d}$ & $3.90 \pm 0.03 \mathrm{de}$ & $.074 \mathrm{~d}$ \\
\hline Half & G & 53.8 & 15.74 & 3.40 & & 5.62 & \\
\hline recommended & A & $46.72 \pm 1.26 c$ & $9.22 \pm 0.62 c$ & $3.80 \pm 0.074 \mathrm{c}$ & $19.14 \pm 0.73 \mathrm{c}$ & $5.80 \pm 0.24 \mathrm{~d}$ & $1.63 \pm 0.21 \mathrm{~b}$ \\
\hline \multirow[t]{3}{*}{ (0.200 g/pot) } & $\mathrm{G}+\mathrm{Pf}$ & & $19.5=$ & & & & $21 \mathrm{a}$ \\
\hline & $\mathrm{A}+\mathrm{Pf}$ & 46.70 & $12.4 \pm 0.54 \mathrm{bc}$ & 3.30 & $22.70 \pm 1.16 \mathrm{~b}$ & $7.22 \pm 0.69 \mathrm{~b}$ & $2.03 \pm 0.23 \mathrm{ab}$ \\
\hline & $\mathrm{G}+\mathrm{A}+\mathrm{Pf}$ & $57.02 \pm 0.96 \mathrm{~b}$ & $23.9 \pm 0.24 \mathrm{ab}$ & $5.56 \pm 0.4 \mathrm{bc}$ & $39.62 \pm 0.86 \mathrm{a}$ & $9.51 \pm 1.02 \mathrm{a}$ & $2.73 \pm 0.38 \mathrm{a}$ \\
\hline F2 & Control & $23.40 \pm 1.42 \mathrm{e}$ & $5.96 \pm 0.4$ & $2.18 \pm 0.4 \mathrm{e}$ & $11.16 \pm 0.64 \mathrm{~d}$ & 3.0 & $0.6 \mathrm{c}$ \\
\hline Recommended & G & $54.50 \pm 1.82 \mathrm{~b}$ & $24.7 \pm 0.77 \mathrm{ab}$ & $5.23 \pm 0.31 \mathrm{~b}$ & $22.42 \pm 1 \mathrm{~b}$ & $5.38 \pm 0.53 d$ & $1.39 \pm 0.06 \mathrm{~b}$ \\
\hline \multirow[t]{4}{*}{$(0.400 \mathrm{~g} / \mathrm{pot}$} & A & 44.88 & $15.8 \pm$ & $3.9=$ & $0.87 \mathrm{c}$ & 5.00 & $1.18 \pm 0.065 b c$ \\
\hline & $\mathrm{G}+\mathrm{Pf}$ & $68.64 \pm 0.58 \mathrm{a}$ & $22.3 \pm 1.16 \mathrm{ab}$ & $5.37 \pm$ & $21.40 \pm 0.8 b c$ & $6.23 \pm$ & $1.33 \pm 0.06 \mathrm{~b}$ \\
\hline & $\mathrm{A}+\mathrm{Pf}$ & $47.10 \pm 2.26 \mathrm{c}$ & $17.6 \pm 0.99 b$ & $3.78 \pm 0.24 c$ & $20.86 \pm 1.14 \mathrm{bc}$ & $6.11 \pm 0.48 c$ & $1.51 \pm 0.12 \mathrm{~b}$ \\
\hline & $\mathrm{G}+\mathrm{A}+\mathrm{Pf}$ & 67.2 & $28.2 \pm$ & 6.08 & $2 \mathrm{bc}$ & & $0.08 \mathrm{ab}$ \\
\hline F3 & Control & $20.00=$ & $6.12 \pm$ & 1.50 & 9.0 .0 & 2.55 & $0.60 \pm 0.05 \mathrm{e}$ \\
\hline Double & G & $25.92 \pm 2.23 \mathrm{e}$ & $7.96 \pm 0.32 \mathrm{~d}$ & $2.36 \pm 0.30 \mathrm{e}$ & $7.62 \pm 0.35 f$ & $2.89 \pm 0.27 \mathrm{f}$ & $0.77 \pm 0.07 \mathrm{de}$ \\
\hline recon & A & $25.16 \pm 2 \mathrm{e}$ & $7.02 \pm 0.19 \mathrm{~d}$ & $2.46 \pm 0.23 \mathrm{e}$ & $6.40 \pm 0.45 \mathrm{f}$ & $3.05 \pm 0.15 \mathrm{e}$ & $0.88 \pm 0.09 \mathrm{~d}$ \\
\hline \multirow[t]{3}{*}{ (0.800 g/pot) } & $\mathrm{G}+\mathrm{Pf}$ & $23.56 \pm 2.28 \mathrm{e}$ & $5.86 \pm 0.44 \mathrm{e}$ & $1.94 \pm 0.12 \mathrm{e}$ & $9.40 \pm 0.51 \mathrm{e}$ & $4.01 \pm 0.30 \mathrm{de}$ & $1.04 \pm 0.13 \mathrm{c}$ \\
\hline & $\mathrm{A}+\mathrm{Pf}$ & $25.66 \pm 1.69 \mathrm{e}$ & $5.18 \pm 0.3$ & 2.12 & $11.0 \pm 0$. & $4.13 \pm 0.52 \mathrm{de}$ & $1.18 \pm 0.14 \mathrm{bc}$ \\
\hline & $\mathrm{G}+\mathrm{A}+1$ & 25.1 & $7.48 \pm$ & $2.60 \pm 0.21 \mathrm{e}$ & $12.5 \pm 0.73 \mathrm{de}$ & $3.93 \pm 0.41 \mathrm{de}$ & $1.22 \pm 0.11 b c$ \\
\hline \multirow{3}{*}{$\begin{array}{l}\text { ANOVA }\left(\mathrm{F}_{5},\right. \\
\left.{ }_{96}\right)\end{array}$} & Treatments & 620.65 & 851.32 & 81.62 & 1937.7 & 305.89 & 359.74 \\
\hline & & 922.71 & 2134.28 & 104.87 & 1676 & 476.22 & 195 \\
\hline & & 292.68 & 175.11 & 31.77 & 1210.6 & 95.46 & 200.52 \\
\hline & & & & & & & \\
\hline & & & & & & & \\
\hline
\end{tabular}

Each value is a mean of five replicates, \pm : standard deviation, G: Glomus mosseae, A: Acaulospora laevis, Pf: Pseudomonas fluorescens, F: Super phosphate fertilization. Means followed by same letter within a column are not significantly different over one another (Duncan's Multiple Range Test, $p \leq 0.05$ ). 
Plants supplied with optimum P fertilizer (F1) are more vigorous, as AM fungal hyphae explore larger soil volume and bridges the gaps between the soil and roots of host plants, making plant stronger and stouter (Smith and Smith 2012). As the chlorophyll content increases in mycorrhizal treated plant there is simultaneous increase in the rate of photosynthesis which can be due to more absorption of nutrients and increase in gaseous exchange by enhanced conductivity of leaf stomata. Without P (F0) as well as increasing $\mathrm{P}$ application at F2 and F3 decreased leaf chlorophyll level, due to reduction in stomatal conductance and hence photosynthesis. On the other hand high fertilizer application built up a stress in the host plants and declines the normal physiological function, whereas AM has been shown to effectively enhance the stomatal conductance,

Table 3. Effect of soil inoculation with AM fungi, P. fluorescens and super phosphate on chlorophyll, photosynthesis and stomatal conductance of bell pepper

\begin{tabular}{|c|c|c|c|c|c|c|}
\hline \multirow{2}{*}{$\begin{array}{l}\text { Super } \\
\text { phosphate } \\
\text { concentration } \\
\text { (g/pot) }\end{array}$} & \multirow{2}{*}{$\begin{array}{l}\text { Parameters } \\
\rightarrow \\
\text { Treatments } \\
\downarrow\end{array}$} & \multicolumn{2}{|c|}{$\begin{array}{l}\text { Chlorophyll content } \\
\left(\mathrm{mg}^{-1} \mathrm{~g}^{-1} \text { FM) }\right.\end{array}$} & \multirow[t]{2}{*}{$\begin{array}{l}\text { Photosynthesis } \\
\mathrm{mgCO}_{2} \mathrm{~m}^{-2} \mathrm{~s}^{-1}\end{array}$} & \multicolumn{2}{|c|}{$\begin{array}{l}\text { Stomatal conductance } \\
\left(\mathrm{mmol}^{-2} \mathbf{s}^{-2}\right)\end{array}$} \\
\hline & & Chlorophyll a & $\begin{array}{l}\text { Chlorophyll } \\
\text { b }\end{array}$ & & Lower & Upper \\
\hline \multirow{6}{*}{$\begin{array}{l}\text { F0 } \\
\text { Without } \\
\text { fertilizer }\end{array}$} & Control & $0.030 \pm 0 \mathrm{f}$ & $0.30 \pm 0.017 \mathrm{de}$ & $17.52 \pm 1.58 \mathrm{~d}$ & $121.2 \pm 2.4 \mathrm{~d}$ & $51.40 \pm 2.8 \mathrm{~d}$ \\
\hline & G & $0.049 \pm 0 \mathrm{de}$ & $0.52 \pm 0.008 \mathrm{c}$ & $34.32 \pm 0.7 b c$ & $250.8 \pm 4.34 \mathrm{~b}$ & $132.4 \pm 5.9 \mathrm{a}$ \\
\hline & A & $0.052 \pm 0.004 \mathrm{~d}$ & $0.53 \pm .007 \mathrm{c}$ & $29.04 \pm 1.6 \mathrm{c}$ & $124.8 \pm 3.1 \mathrm{~d}$ & $63.40 \pm 3.8 \mathrm{~cd}$ \\
\hline & $\mathrm{G}+\mathrm{Pf}$ & $0.058 \pm 0.004 \mathrm{~cd}$ & $0.61 \pm 0.006 \mathrm{bc}$ & $39.48 \pm 1.02 \mathrm{bc}$ & $252.8 \pm 3.84 \mathrm{~b}$ & $108.2 \pm 2.2 \mathrm{ab}$ \\
\hline & $A+P f$ & $0.047 \pm 0.004 \mathrm{de}$ & $0.52 \pm 0 \mathrm{c}$ & $27.84 \pm 4.42 c$ & $268.0 \pm 2.55 \mathrm{~b}$ & $134.2 \pm 2.9 \mathrm{a}$ \\
\hline & $\mathrm{G}+\mathrm{A}+\mathrm{Pf}$ & $0.039 \pm 0.004 \mathrm{e}$ & $0.44 \pm 0.01 \mathrm{~d}$ & $44.92 \pm 1.78 \mathrm{~b}$ & $199.0 \pm 3.4 b c$ & $85.00 \pm 1.82 \mathrm{bc}$ \\
\hline \multirow{6}{*}{$\begin{array}{l}\text { F1 } \\
\text { Half } \\
\text { recommended } \\
(0.200 \mathrm{~g} / \mathrm{pot})\end{array}$} & Control & $0.066 \pm 0.055 \mathrm{c}$ & $0.23 \pm 0.009 \mathrm{e}$ & $20.06 \pm 0.40 \mathrm{~d}$ & $94.64 \pm 1.44 \mathrm{de}$ & $43.16 \pm 1.72 \mathrm{e}$ \\
\hline & G & $0.090 \pm 0.01 b c$ & $0.78 \pm 0.013 b$ & $37.46 \pm 0.73 b c$ & $139.5 \pm 3.24 c$ & $73.92 \pm 1.08 \mathrm{c}$ \\
\hline & A & $0.130 \pm 0.007 \mathrm{~b}$ & $0.41 \pm 0.0084 \mathrm{~d}$ & $35.04 \pm 1.02 \mathrm{bc}$ & $154.2 \pm 1.5 \mathrm{c}$ & $74.36 \pm 1.44 \mathrm{c}$ \\
\hline & $\mathrm{G}+\mathrm{Pf}$ & $0.254 \pm 0.011 \mathrm{a}$ & $0.71 \pm 0.0084 b$ & $38.42 \pm 1.31 b c$ & $199.4 \pm 2.6 b c$ & $73.92 \pm 1.08 \mathrm{c}$ \\
\hline & $\mathrm{A}+\mathrm{Pf}$ & $0.158 \pm 0.0084 \mathrm{~b}$ & $0.47 \pm 0.0084 d$ & $33.88 \pm 1.12 b c$ & $235.6 \pm 2.02 b$ & $97.48 \pm 1.62 b$ \\
\hline & $\mathrm{G}+\mathrm{A}+\mathrm{Pf}$ & $0.272 \pm 0.0084 \mathrm{a}$ & $0.83 \pm 0.009 \mathrm{a}$ & $51.06 \pm 2.96 \mathrm{a}$ & $448.5 \pm 4.75 \mathrm{a}$ & $123.0 \pm 2.4 \mathrm{a}$ \\
\hline \multirow{6}{*}{$\begin{array}{l}\text { F2 } \\
\text { Recommended } \\
(0.400 \mathrm{~g} / \text { pot }\end{array}$} & Control & $0.048 \pm 0.005 \mathrm{de}$ & $0.19 \pm 0.0084 \mathrm{e}$ & $19.86 \pm 2.71 \mathrm{~d}$ & $80.88 \pm 1.43 \mathrm{e}$ & $29.32 \pm 1.9 \mathrm{f}$ \\
\hline & G & $0.050 \pm 0.007 \mathrm{~d}$ & $0.44 \pm 0.005 \mathrm{~d}$ & $27.00 \pm 1.75 \mathrm{c}$ & $123.6 \pm 3.6 \mathrm{~d}$ & $62.30 \pm 1.85 \mathrm{cc}$ \\
\hline & A & $0.040 \pm 0 \mathrm{de}$ & $0.37 \pm 0.005 \mathrm{de}$ & $27.96 \pm 1.1 \mathrm{c}$ & $188.4 \pm 5.8 \mathrm{bc}$ & $93.06 \pm 1.51 \mathrm{~b}$ \\
\hline & $\mathrm{G}+\mathrm{Pf}$ & $0.088 \pm 0.084 b c$ & $0.53 \pm 0.0084 c$ & $27.58 \pm 1.83 \mathrm{c}$ & $124.1 \pm 3.2 \mathrm{~d}$ & $62.30 \pm 1.85 \mathrm{~cd}$ \\
\hline & $A+P f$ & $0.050 \pm 0 \mathrm{~d}$ & $0.43 \pm 0.02 \mathrm{~d}$ & $28.96 \pm 1.25 \mathrm{c}$ & $158.3 \pm 3.35 c$ & $79.50 \pm 2.52 b c$ \\
\hline & $\mathrm{G}+\mathrm{A}+\mathrm{Pf}$ & $0.140 \pm 0.01 \mathrm{~b}$ & $0.53 \pm 0.02 \mathrm{c}$ & $29.40 \pm 1.84 \mathrm{c}$ & $228.1 \pm 4.8 \mathrm{~b}$ & $82.72 \pm 4.74 \mathrm{bc}$ \\
\hline \multirow{6}{*}{$\begin{array}{l}\mathrm{F} 3 \\
\text { Double } \\
\text { recommended } \\
(0.800 \mathrm{~g} / \text { pot })\end{array}$} & Control & $0.066 \pm 0.011 \mathrm{c}$ & $0.22 \pm 0.016 \mathrm{e}$ & $16.38 \pm 0.84 \mathrm{~d}$ & $66.82 \pm 3.68 \mathrm{f}$ & $38.12 \pm 2.85 \mathrm{e}$ \\
\hline & G & $0.048 \pm 0.004 \mathrm{de}$ & $0.18 \pm 0.0084 \mathrm{e}$ & $14.82 \pm 1.01 \mathrm{e}$ & $87.10 \pm 1.02 \mathrm{e}$ & $34.38 \pm 4.43 \mathrm{ef}$ \\
\hline & A & $0.020 \pm 0.007 \mathrm{f}$ & $0.09 \pm 0.007 \mathrm{f}$ & $14.24 \pm 1.42 \mathrm{e}$ & $55.90 \pm 3.47 \mathrm{~g}$ & $32.14 \pm 5.65 \mathrm{ef}$ \\
\hline & $\mathrm{G}+\mathrm{Pf}$ & $0.050 \pm 0 \mathrm{~d}$ & $0.23 \pm 0.013 \mathrm{e}$ & $16.20 \pm 1.14 \mathrm{~d}$ & $74.80 \pm 3.01 \mathrm{ef}$ & $40.80 \pm 2.84 \mathrm{e}$ \\
\hline & $\mathrm{A}+\mathrm{Pf}$ & $0.040 \pm 0.007 \mathrm{de}$ & $0.12 \pm 0.007 \mathrm{f}$ & $18.18 \pm 1.08 \mathrm{~d}$ & $64.74 \pm 3.76 f$ & $25.33 \pm 2.38 \mathrm{f}$ \\
\hline & $\mathrm{G}+\mathrm{A}+\mathrm{Pf}$ & $0.050 \pm 0.007 \mathrm{~d}$ & $0.21 \pm 0.0075 \mathrm{e}$ & $18.60 \pm 1.31 \mathrm{~d}$ & $82.30 \pm 7.72 \mathrm{e}$ & $58.34 \pm 5 \mathrm{~d}$ \\
\hline \multirow{2}{*}{$\begin{array}{l}\text { ANOVA }\left(\mathrm{F}_{5},\right. \\
\left.{ }_{96}\right)\end{array}$} & Treatments & 562.65 & 175.5 & 296.12 & 426.38 & 585.35 \\
\hline & (t) & 1824.04 & 528.24 & 58.42 & 73.16 & 136.26 \\
\hline \multirow{3}{*}{$\begin{array}{l}\left(\mathrm{F}_{3},\right. \\
96) \quad\left(\mathrm{F}_{15},\right.\end{array}$} & Fertilizer & 213.53 & 701.0 & 45.32 & 154.56 & 280 \\
\hline & & & & & & \\
\hline & $t \times F$ & & & & & \\
\hline
\end{tabular}

Each value is a mean of five replicates, \pm : standard deviation, G: Glomus mosseae, A: Acaulospora laevis, Pf: Pseudomonas fluorescens, F: Super phosphate fertilization. Means followed by same letter within a column are not significantly different over one another (Duncan's Multiple Range Test, $p \leq 0.05$ ). 
photosynthetic rate and water use efficiency of their host during severe stress conditions (Querejeta et al., 2006).

\subsection{Effect on nutrition}

Mycorrhizal inoculation alone did not significantly influence the concentration of plant phosphorus and total nitrogen $(\mathrm{N})$. However, AM fungi and $\mathrm{P}$ fertilizer together resulted in significant increase in the concentration of both phosphorus and nitrogen compared to their respective control but higher P application doesn't support the increment in plant nutrient level (Table 4). These results are in accordance with the findings of Rakshit and Bhadoria (2009) that highest phosphorus uptake occurs in mycorrhizal maize plants with added low $\mathrm{P}$ than non-mycorrhizal as well as plants without added P. Mycorrhizal inoculation consistently accumulated more quantities of phosphorus in their root than shoots.

Table 4. Effect of soil inoculation with AM fungi, P. fluorescens and super phosphate on $\mathrm{P}$ and $\mathrm{N}$ nutrition of green bell pepper

\begin{tabular}{|c|c|c|c|c|}
\hline \multirow{2}{*}{$\begin{array}{l}\text { Super phosphate } \\
\text { concentration (g/pot) }\end{array}$} & \multirow{2}{*}{$\begin{array}{l}\text { Parameters } \rightarrow \\
\text { Treatments } \downarrow\end{array}$} & \multicolumn{2}{|c|}{ Plant phosphorus content (\%) } & \multirow[t]{2}{*}{ Total nitrogen content $(\%)$} \\
\hline & & Shoot & Root & \\
\hline F1 & Control & $0.28 \pm 0.019 \mathrm{f}$ & $0.34 \pm 0.056 \mathrm{f}$ & $3.23 \pm 0.26 \mathrm{e}$ \\
\hline \multirow{5}{*}{$\begin{array}{l}\text { Half recommended } \\
(0.300 \mathrm{~g} / \text { pot })\end{array}$} & $\mathrm{G}$ & $0.40 \pm 0.011 \mathrm{~d}$ & $0.46 \pm 0.005 \mathrm{~cd}$ & $4.71 \pm 0.09 \mathrm{~b}$ \\
\hline & A & $0.37 \pm 0.027 \mathrm{de}$ & $0.44 \pm 0.004 \mathrm{~cd}$ & $4.4 \pm 0.13 c$ \\
\hline & $\mathrm{G}+\mathrm{Pf}$ & $0.31 \pm 0.03 \mathrm{a}$ & $0.79 \pm 0.005 \mathrm{a}$ & $5.3 \pm 0.08 \mathrm{a}$ \\
\hline & $\mathrm{A}+\mathrm{Pf}$ & $0.51 \pm 0.025 b$ & $0.66 \pm 0.011 b$ & $4.62 \pm 0.08 b$ \\
\hline & $\mathrm{G}+\mathrm{A}+\mathrm{Pf}$ & $0.59 \pm 0.031 \mathrm{a}$ & $0.77 \pm 0.005 \mathrm{a}$ & $5.13 \pm 0.09 \mathrm{a}$ \\
\hline F2 & Control & $0.31 \pm 0.005 \mathrm{ef}$ & $0.37 \pm 0.030 \mathrm{e}$ & $3.04 \pm 0.16 \mathrm{e}$ \\
\hline Recommended & $\mathrm{G}$ & $0.38 \pm 0.023 \mathrm{de}$ & $0.45 \pm 0.007 \mathrm{~cd}$ & $3.08 \pm 0.16 \mathrm{e}$ \\
\hline \multirow[t]{4}{*}{$(0.600 \mathrm{~g} / \mathrm{pot}$} & $\mathrm{A}$ & $0.36 \pm 0.012 \mathrm{e}$ & $0.38 \pm 0.005 \mathrm{e}$ & $4.1 \pm 0.13 \mathrm{c}$ \\
\hline & $\mathrm{G}+\mathrm{Pf}$ & $0.43 \pm 0.016 \mathrm{~d}$ & $0.48 \pm 0.009 \mathrm{~cd}$ & $3.7 \pm 0.18 \mathrm{~d}$ \\
\hline & $\mathrm{A}+\mathrm{Pf}$ & $0.41 \pm 0.016 \mathrm{c}$ & $0.53 \pm 0.004 \mathrm{c}$ & $4.37 \pm 0.10 \mathrm{c}$ \\
\hline & $\mathrm{G}+\mathrm{A}+\mathrm{Pf}$ & $0.5 \pm 0.029 b$ & $0.67 \pm 0.012 \mathrm{~b}$ & $4.1 \pm 0.14 \mathrm{c}$ \\
\hline F3 & Control & $0.23 \pm 0.01 \mathrm{f}$ & $0.35 \pm 0.013 \mathrm{f}$ & $3.25 \pm 0.32 \mathrm{e}$ \\
\hline Double recommended & $\mathrm{G}$ & $0.37 \pm 0.013 \mathrm{de}$ & $0.37 \pm 0.01 \mathrm{e}$ & $3.25 \pm 0.32 \mathrm{e}$ \\
\hline \multirow[t]{4}{*}{$(1.200 \mathrm{~g} / \mathrm{pot})$} & A & $0.28 \pm 0.022 \mathrm{f}$ & $0.34 \pm 0.013 \mathrm{f}$ & $3.14 \pm 0.3 \mathrm{e}$ \\
\hline & $\mathrm{G}+\mathrm{Pf}$ & $0.37 \pm 0.036 \mathrm{de}$ & $0.48 \pm 0.065 \mathrm{~cd}$ & $2.68 \pm 0.18 \mathrm{f}$ \\
\hline & $\mathrm{A}+\mathrm{Pf}$ & $0.3 \pm 0.016 \mathrm{de}$ & $0.41 \pm 0.015 \mathrm{~d}$ & $3.56 \pm 0.26 \mathrm{~d}$ \\
\hline & $\mathrm{G}+\mathrm{A}+\mathrm{Pf}$ & $0.41 \pm 0.026 \mathrm{c}$ & $0.51 \pm 0.013 \mathrm{c}$ & $3.07 \pm 0.2 \mathrm{e}$ \\
\hline $\operatorname{ANOVA}\left(\mathrm{F}_{5}, 96\right)$ & Treatments (t) & 316.89 & 1981.4 & 120.6 \\
\hline$\left(\mathrm{F}_{3}, 96\right)$ & Fertilizer $(\mathrm{F})$ & 582.86 & 1385.2 & 541.6 \\
\hline$\left(\mathrm{F}_{15}, 96\right.$ & $\mathrm{t} \times \mathrm{F}$ & 17.8 & 224.75 & 33.66 \\
\hline
\end{tabular}

Each value is a mean of five replicates, \pm : standard deviation, G: Glomus mosseae, A: Acaulospora laevis, Pf: Pseudomonas fluorescens, F: Super phosphate fertilization. Means followed by same letter within a column are not significantly different over one another (Duncan's Multiple Range Test, $p \leq 0.05$ ). 
After 120 days of inoculation, the increase in phosphorus content in shoot was found maximum in plants treated with $\mathrm{G}+\mathrm{A}+\mathrm{Pf}(0.59 \pm 0.031)$ and for root in $\mathrm{G}+\mathrm{Pf}(0.79 \pm 0.005)$ at $\mathrm{F} 1$ as compared to uninoculated control. As shown in Table 4 it is clear that soil inoculation with arbuscular mycorrhizal fungi and $P$. fluorescens along with different doses of superphosphate fertilizer markedly improved the total $\mathrm{N} \%$ in bell pepper plants in comparison to uninoculated control. The highest increase in total $\mathrm{N} \%$ was observed in $\mathrm{G}+\mathrm{Pf}(5.3 \pm 0.08)$ of $\mathrm{F} 1$. The increase in the nutrient content of the plants inoculated with Pf is mainly due to the changes produced in the root morphology by phytohormones synthesized, that results in an increase in root surface area (Bashan et al., 2004).

Table 5. Effect of soil inoculation with AM fungi, P. fluorescens and super phosphate on yield of bell pepper plants

\begin{tabular}{|c|c|c|c|c|c|}
\hline $\begin{array}{l}\text { Super phosphate } \\
\text { concentration } \\
\text { (g/pot) }\end{array}$ & $\begin{array}{l}\text { Parameters } \\
\rightarrow \\
\text { Treatments } \downarrow\end{array}$ & $\begin{array}{l}\text { No. of days } \\
\text { of flowering }\end{array}$ & $\begin{array}{l}\text { No. of days } \\
\text { of fruiting }\end{array}$ & $\begin{array}{l}\text { No. of } \\
\text { fruits/plant }\end{array}$ & $\begin{array}{l}\text { Fresh weigh } \\
\text { of fruits } \\
\text { (g)/plant }\end{array}$ \\
\hline F0 & Control & of & of & of & $0 \mathrm{~h}$ \\
\hline Without & G & $79.8 \pm 1.48 \mathrm{~b}$ & $89.80 \pm 1.1 \mathrm{~b}$ & $1.8 \pm 0.45 c$ & $4.80 \pm 0.4 \mathrm{~d}$ \\
\hline \multirow[t]{4}{*}{ fertilizer } & A & $85.4 \pm 1.82 \mathrm{c}$ & $94.60 \pm 1.34 \mathrm{c}$ & $1.6 \pm 0.55 \mathrm{~cd}$ & $2.23 \pm 0.46 \mathrm{e}$ \\
\hline & $\mathrm{G}+\mathrm{Pf}$ & $69.4 \pm 1.67 \mathrm{a}$ & $78.20 \pm 2.1 \mathrm{a}$ & $2.8 \pm 0.45 b$ & $18.4 \pm 0.71 b$ \\
\hline & $A+P f$ & $88.6 \pm 2.4 \mathrm{bc}$ & $108.0 \pm 5.45 \mathrm{~d}$ & $1.8 \pm 0.45 c$ & $4.25 \pm 0.87 \mathrm{~d}$ \\
\hline & $\mathrm{G}+\mathrm{A}+\mathrm{Pf}$ & $79.6 \pm 1.82 \mathrm{~b}$ & $87.60 \pm 2.7 \mathrm{~b}$ & $3.2 \pm 0.45 \mathrm{ab}$ & $11.9 \pm 0.60 \mathrm{c}$ \\
\hline F1 & Control & $95.0 \pm 1.67 \mathrm{~cd}$ & $94.80 \pm 1.79 \mathrm{c}$ & of & $0 \mathrm{~h}$ \\
\hline Half & G & $79.6 \pm 1.14 \mathrm{~b}$ & $92.83 \pm 3.49 \mathrm{c}$ & $1.8 \pm 0.45 c$ & $9.24 \pm 0.39 c$ \\
\hline recommended & A & $84.6 \pm 1.1 \mathrm{c}$ & $96.60 \pm 1.14 \mathrm{c}$ & $1.8 \pm 0.45 c$ & $4.84 \pm 0.40 \mathrm{~d}$ \\
\hline \multirow[t]{3}{*}{$(0.200 \mathrm{~g} / \mathrm{pot})$} & $\mathrm{G}+\mathrm{Pf}$ & $76.0 \pm 1.87 \mathrm{~b}$ & $89.30 \pm 1.58 \mathrm{~b}$ & $3.8 \pm 0.45 \mathrm{a}$ & $14.2 \pm 1.12 \mathrm{bc}$ \\
\hline & $\mathrm{A}+\mathrm{Pf}$ & $82.8 \pm 0.84 \mathrm{c}$ & $93.60 \pm 1.82 \mathrm{c}$ & $2.4 \pm 0.55 b$ & $11.6 \pm 0.48 c$ \\
\hline & $\mathrm{G}+\mathrm{A}+\mathrm{Pf}$ & $67.6 \pm 1.82 \mathrm{a}$ & $84.80 \pm 1.92 \mathrm{ab}$ & $3.6 \pm 0.55 a$ & $26.4 \pm 2.2 \mathrm{a}$ \\
\hline $\mathrm{F} 2$ & Control & $90.0 \pm 1 \mathrm{c}$ & $90.40 \pm 0.89 \mathrm{~b}$ & of & $0 \mathrm{~h}$ \\
\hline Recommended & G & $87.6 \pm 1.59 \mathrm{c}$ & $96.40 \pm 0 \mathrm{c}$ & $0.6 \pm 0.55 \mathrm{e}$ & $2.06 \pm 1.92 \mathrm{f}$ \\
\hline \multirow[t]{4}{*}{$(0.400 \mathrm{~g} / \mathrm{pot}$} & A & $105 . \pm 1.92 \mathrm{~d}$ & $116.5 \pm 1.12 \mathrm{e}$ & $0.4 \pm 0.55 \mathrm{e}$ & $0.43 \pm 0.6 \mathrm{~g}$ \\
\hline & $\mathrm{G}+\mathrm{Pf}$ & $75.4 \pm 1.52 \mathrm{~b}$ & $93.80 \pm 1.3 \mathrm{c}$ & $2.6 \pm 0.55 b$ & $9.06 \pm 0.69 c$ \\
\hline & $\mathrm{A}+\mathrm{Pf}$ & $96.4 \pm 1.14 \mathrm{a}$ & $111.0 \pm 2.65 b$ & $1.0 \pm 1 \mathrm{~d}$ & $1.99 \pm 1.81 \mathrm{f}$ \\
\hline & $\mathrm{G}+\mathrm{A}+\mathrm{Pf}$ & $84.0 \pm 1 \mathrm{c}$ & 95.55 & $2.6 \pm 0.55 b$ & $14.9 \pm 1.2 b c$ \\
\hline F3 & Control & $90.0 \pm 1.66 \mathrm{c}$ & of & of & $0 \mathrm{~h}$ \\
\hline Double & G & $113 \pm 1.67 \mathrm{e}$ & of & of & $0 \mathrm{~h}$ \\
\hline recommended & A & of & of & of & $0 \mathrm{~h}$ \\
\hline \multirow[t]{3}{*}{ (0.800 g/pot) } & $\mathrm{G}+\mathrm{Pf}$ & $102 \pm 1.48 \mathrm{~d}$ & of & of & $0 \mathrm{~h}$ \\
\hline & $\mathrm{A}+\mathrm{Pf}$ & of & of & of & $0 \mathrm{~h}$ \\
\hline & $\mathrm{G}+\mathrm{A}+\mathrm{Pf}$ & $106 \pm 1.48 \mathrm{~d}$ & $112.6 \pm 1.95 \mathrm{e}$ & $1.4 \pm 0.55 \mathrm{~cd}$ & $1.8 \pm 0.5 \mathrm{f}$ \\
\hline \multirow{3}{*}{$\begin{array}{r}\operatorname{ANOVA}\left(\mathrm{F}_{5}, 96\right) \\
\left(\mathrm{F}_{3}, 96\right) \\
\left(\mathrm{F}_{15}, 96\right.\end{array}$} & Treatments & 862.02 & 49.85 & 158.2 & 398.78 \\
\hline & $(\mathrm{t})$ & 1453.87 & 32.77 & 34.2 & 731.38 \\
\hline & $\begin{array}{l}\text { Fertilizer }(F) \\
t \times F\end{array}$ & 2979.49 & 9.73 & 6.51 & 105.74 \\
\hline
\end{tabular}

Each value is a mean of five replicates, \pm : standard deviation, G: Glomus mosseae, A: Acaulospora laevis, Pf: Pseudomonas fluorescens, F: Super phosphate fertilization. Means followed by same letter within a column are not significantly different over one another (Duncan's Multiple Range Test, $p \leq 0.05$ ). 
At the same time there is an increase in the activities of the acid and alkaline phosphates enzymes produced by the plant roots itself, extraradical hyphae of AM fungi as well as by $P$. fluorescens that play an important role in the cycling of phosphorus from $\mathrm{P}$ deficient soils and helps in the phosphorus nutrition of plants (Tanwar et al., 2012). Moreover, the AM fungi colonized roots uses the extraradical mycelium to explore a greater volume of soil, and translocate nutrients from soil to the plants more efficiently, resulting in better and improved plant nutrition (Linderman, 1992). The enhanced growth and nutritional status of bell pepper is also related to the percent root colonization apart from several soil and environmental conditions as maximum colonization as well as $\mathrm{P}$ content was recorded in the same treatment $\mathrm{G}+\mathrm{A}+\mathrm{Pf}$ at $\mathrm{F} 1$.

\subsection{Effect on yield}

Early flowering and fruit formation was recorded as a result of inoculation with efficient strains. Flowering was first observed in the treatment of $\mathrm{G}+\mathrm{A}+\mathrm{Pf}(67.6 \pm 1.82)$ at $\mathrm{F} 1 \mathrm{P}$ followed by $\mathrm{G}+\mathrm{Pf}$ $(76 \pm 1.87)$ at $\mathrm{F} 0$ (Table 5). However, fruit formation first appeared in $\mathrm{G}+\mathrm{Pf}(78.2 \pm 2.1)$ at $\mathrm{F} 0$ followed by $\mathrm{G}+\mathrm{A}+\mathrm{Pf}(84.8 \pm 1.92)$ at $\mathrm{F} 1$.

Fruit formation doesn't occur in some treatments of F3 fertilized pots and in uninoculated control plants. This can be due to low AM colonization level and lesser nutrients accumulation in these plants. At higher $\mathrm{P}$ levels, malformation of apical tip was recorded that results in stunted growth. Increase in the soil $\mathrm{P}$ is known to suppress the growth and availability of AM fungi in the soil by reducing root colonization and also spore density (Fusconi et al., 2005). High P supply had little or no effect on fruit yield despite the low mycorrhizal colonization of the roots by native endophytes. In contrast to this, mycorrhizal inoculation resulted in significant increase in fruit yield of bell pepper at F1.

Multi inoculation of $\mathrm{G}+\mathrm{A}+\mathrm{Pf}$ synergistically increased fruit number $(3.8 \pm 0.45)$ and fruit weight
(26.38 \pm 2.2$)$. These results are in close conformity with other workers that root inoculation of bell pepper with AM fungi or rhizobacteria significantly increases fruit yield (Constantino et al., 2008; Tanwar et al., 2012; Tanwar et al., 2013). This may result from mycorrhizal enhancement of plant growth and development, which along with $P$. fluorescens increased $\mathrm{P}$ absorption and translocation to other parts of the plant (Tanwar et al., 2012). De Giorgio et al. (2004) also reported that lower dose of fertilizer (N2) enhances grain yield of durum wheat when inoculated with AM fungi than highly fertilized and unfertilized plants.

Reduction in fertilizer input on application of beneficial microbes has also been reported by Soleimanzadeh (2010) who suggested use of AM fungi with 50 percent recommended phosphorus to increase seed yield and oil production in sunflower.

\section{Conclusion}

The present study demonstrated that the dual inoculation and multi inoculation of bell pepper plants with G. mosseae, A. laevis and P. fluorescens increased growth, photosynthetic rate, fruit number, fruit weight, total nitrogen content and phosphorus content at F1, below the recommended supplied $\mathrm{P}$ over unfertilized as well as heavy fertilized plants by enhanced bioavailability and mobility of plant nutrients. Increased P supply increased some parameters connected to plant height, shoot fresh and dry weight but at same time decreased percent of mycorrhizal colonization and hence $\mathrm{P}$ content and fruit yield. Thus, soil amendment with AM fungi and P. fluorescens have the potential to possibly reduce the application of phosphorus fertilizer for crop improvement, growth, yield and nutritional value of Capsicum annuum for bell pepper growers.

\section{Acknowledgements}

The author (AT) is grateful to Kurukshetra University, 
Kurukshetra, for providing financial assistance in the form of URS (University Research Fellowship) to carry out this investigation. The authors are also indebted to Dr. Hardeep Joshi, Department of Psychology, Kurukshetra University, Kurukshetra for his help in statistical analysis of the data.

\section{References}

Anguilera-Gomez, L., Davies, F.T., Olalde-Portugal, V., Duray, S.A., Phavaphutanon, L. 1999. Influence of phosphorus and endomycorrhiza (Glomus intraradices) on gas exchange and plant growth of chile ancho pepper (Capsicum annuum L. cv. San Luis). Photosynthetica, 36, 3, 441-449.

Arnon, D.L., 1949. A copper enzyme is isolated chloroplast polyphenol oxidase in Beta vulgaries. Plant Physiol. 24, 1-15.

Bashan, Y., Holguin, G., De Bashan, L.E. 2004. Azospirillum-plant relationship: physiological, molecular, agriculture and environmental advances (1997-2003). Can. J. Microbiol. 50, 521-577.

Boer, W.D., L.B. Folman, R.C. Summerbell, L. Boddy. 2005. Living in a fungal world: Impact of fungi on soil bacterial niche development. FEMS Microbiol. Rev. 29, 795-811.

Constantino, M., Gómez-Álvarez, R., Álvarez-Solís, J.D., Geissen, V., Huerta, E. Barba, E. 2008. Effect of inoculation with rhizobacteria and Arbuscular Mycorrhizal fungi on growth and yield of Capsicum chinense Jacquin. J. Agric. Rural Dev. Trop. Subtrop. 109, 2, 169-180.

Covacevich, F., Echeverria, H.E. 2008. Receptivity of an Argentinean pampas soil to arbuscular mycorrhizal Glomus and Acaulospora strain. World J. Agric. Sci. 4, 688-698.
De Giorgio, D., Sisto, D., Ubaldo, D., Vonella, A.V. 2008. The effect of $\mathrm{N}$-fertilization levels and root mycorrhizal colonization on plant-growth and grain yield in durum wheat. 13th International Soil Conservation Organization Conference-Brisbane, July 2004. Conserving Soil and Water for Society: Sharing Solutions, pp: 762.

Eslamyan, L., Alipour, Z.T., Beidokhty, S.R., Sobhanipour, A. 2013. Pseudomonas fluorescens and sulfur application affect rapeseed growth and nutrient uptake in calcareous soil. Intl. J. Agri. Crop Sci. 5,1, 39-43.

Fusconi, A., Lingua, G., Trotta, A., Berta, G. 2005. Effects of arbuscular mycorrhizal colonization and phosphorus application on nuclear ploidy in Allium porrum plants. Mycorrhiza, 15, 313-321.

Gamalero, E., Trotta, A., Massa, N., Copetta, A., Martinotti, M.G., Berta, G. 2004. Impact of two fluorescent pseudomonads and an arbuscular mycorrhizal fungus on tomato plant growth, root architecture and $\mathrm{P}$ acquisition. Mycorrhiza, 14, 185-192.

Gamalero, E., Trotta, A., Massa, N., Glick, B.R., Lengua, G. 2008. Synergistic interactions between the ACC deaminase-producing bacterium Pseudomonas putida UW4 and the AM fungus Gigaspora rosea positively affect cucumber plant growth. FEMS Microbiol. Ecol. 64, 459-467.

Gerdemann, J.W., Nicolson, Y.H. 1963. Spores of mycorrhiza Endogone species extracted from soil by wet sieving and decanting. Trans. Brit. Mycol. Soc. 46, 235-244.

Jackson, M.L. 1973. Soil Chemical Analysis Prentice Hall, New Delhi. pp: 485.

Kapoor, K.K., Misra, M.M., Kukreja, K. 1989. Phosphate solubilization by soil microorganisms: A review. Indian J. Microbiol. 29, 119-127. 
Kennedy, I.R., Choudhury, A.T.M.A., Kecskés, M.L. 2004. Non-symbiotic bacterial diazotrophs in crop-farming systems: can their potential for plant growth promotion is better exploited?. Soil Biol. Biochem. 36, 1229-1244.

Linderman, R.G. 1992. Vesicular-arbuscular mycorrhizae and soil microbial interactions. In: Micorrhizae in sustainable agriculture. Bethlenfalvay, G.J. and R.G. Linderman, (eds.), ASA special Publication 54, American Society of Agronomy, Madison, pp: 45-70.

Oke, M., Ahn, T., Schofield, A., Paliyath, G. 2005. Effects of phosphorus fertilizer supplementation on processing quality and functional food ingredients in tomato. J. Agric. Food Chem. 53, 1531-301.

Parkash V, Sharma S, Aggarwal A. 2011. Symbiotic and synergistic efficacy of endomycorrhizae with Dendrocalamus strictus L. Plant Soil Environ. 57, $10,447-452$.

Phillips, J.M., Hayman, D.S. 1970. Improved procedures for clearing roots and staining parasitic and vesicular-arbuscular mycorrhizal fungi for rapid assessment of infection. Trans. Br. Mycol. Soc. $55,159-161$.

Prasad, K., Aggarwal, A. Yadav, K., Tanwar, A. 2012. Impact of different levels of superphosphate using arbuscular mycorrhizal fungi and Pseudomonas fluorescens on Chrysanthemum indicum L. J. Soil Sci. Plant Nutr. 12, 3, 451-462.

Qiao, G., Wen, X.P., Yu, L.F., Ji, X.B. 2011. The enhancement of drought tolerance for pigeon pea inoculated by arbuscular mycorrhizae fungi. Plant Soil Environ. 57, 12, 541-546.

Querejeta, J.I., Allen, M.F., Caravaca, F., Roldan, A. 2006. Differential modulation of host plant delta $\mathrm{C} 13$ and delta $\mathrm{O}-18$ by native and non native arbuscular mycorrhizal fungi in a semi arid environment. New Phytol. 169, 379-387.

Rakshit, A., Bhadoria, P.S. 2009. Role of VAM on growth and phosphorus nutrition of maize with low soluble phosphate fertilization. Acta Agron. $59,1,119-123$.

Schnepf, A., Rose, T., Schweiger, P. 2009. Impact of growth and uptake patterns of arbuscular mycorrhizal fungi on plant phosphorus uptake-a modeling study. Plant Soil. 312, 85-99.

Smith, S.E, Smith, F.A. 2012. Fresh perspectives on the roles of arbuscular mycorrhizal fungi in plant nutrition and growth. Mycologia. 104, 1, 1-13.

Smith, S.E., Read, D.J. 2008. Mycorrhizal symbiosis, 3rd edn. Academic Press, London. Academic Press.

Soleimanzadeh, H. 2010. Effect of VA-Mycorrhiza on growth and yield of Sunflower (Helianthus annuus L.) at different phosphorus levels. World Acad. Sci. Eng. Technol. 71, 414-417.

Tanwar A, Aggarwal A, Karishma, Neetu. 2012. Effectiveness of endomycorrhizal fungi and Pseudomonas fluorescens under different phosphorus levels on Capsicum annuиm L. Kasetsart J. Nat. Sci. 46, 769-782.

Tanwar, A., Aggarwal, A., Alpa, Kadiyan, N. 2011. Enhanced growth and yield of Capsicum annuum L. with two Endomycorrhizal fungi and other bioinoculants. J. Indian Bot. Soc. 90, $351-359$.

Tanwar, A., Aggarwal, A. 2013. Multifaceted potential of bioinoculants on red bell pepper (F1 hybrid, Indam Mamatha) production. J. Plant Interact. DOI:10.1080/17429145.2013.7 65044. 